\title{
Nanoscale
}

D) Check for updates

Cite this: DOI: $10.1039 / \mathrm{c} 8 \mathrm{nr} 09922 \mathrm{~g}$

\section{Direct imaging of dopant sites in rare-earth element-doped permanent magnet and correlated magnetism origin $\dagger$}

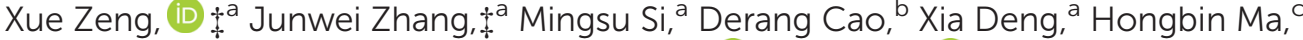 \\ Qianqian Lan, ${ }^{a}$ Desheng Xue, ${ }^{a}$ Xixiang Zhang, (D)*d Kun Tao (D)*a and Yong Peng*a
}

Identifying the dopants and their occupation sites in rare-earth-doped permanent magnets is critical not only to understand the mechanism of tuning their magnetic properties, but also to develop guiding principles to further improve their performance. In this study, we present a direct observation of the preferred atomic sites of $\mathrm{La}$ atoms in $\mathrm{La}$-doped $\mathrm{M}$-type $\mathrm{SrFe}_{12} \mathrm{O}_{19}$ hexaferrite. Our data solidly clarified that only the $\mathrm{Sr}^{2+}$ cations were replaced by $\mathrm{La}^{3+}$ cations, and the La-doping caused the changes in the valence states of iron cations located at the $4 f_{1}$ and 2 a crystallographic sites. First principles calculations further revealed that after La-doping, the changes in the spin states of the $\mathrm{Fe}^{3+}$ cations located at the $4 \mathrm{f}_{1}$ tetrahedral sites resulted in magnetization enhancement and those of the $2 a$ octahedral sites contributed to electrical neutrality, well matching the experimental atomic-column resolution EELS and magnetic measurement results.

Received 7th December 2018

Accepted 9th February 2019

DOI: $10.1039 / \mathrm{c} 8 \mathrm{nr} 09922 \mathrm{~g}$

rsc.li/nanoscale have been many attempts to reduce their cost and further improve their magnetic performances. Among them, slight doping of rare-earth elements has proven to be an effective way. It has been found that varying the dopants and their sites can effectively tailor the electronic configuration and atomic magnetism of M-type ferrites, which lead to significant modification of their magnetic performance. These modulations have been recently demonstrated to have an extended application in multiferroic spintronics because of the additional contribution of physical freedom. ${ }^{8}$ Diverse state-of-art techniques, including Mössbauer spectroscopy ${ }^{9,10}$ neutron diffraction, ${ }^{11}$ X-ray absorption spectroscopy (XAS), ${ }^{12,13}$ X-ray spectroscopy, ${ }^{14}$ X-ray magnetic circular dichroism (XMCD), ${ }^{13}$ nuclear magnetic resonance $(\mathrm{NMR})^{15}$ and Raman spectroscopy ${ }^{16}$ have been employed to detect the sites of the dopants and the ordering of cations. However, due to the limitation of the wavelengths of their light sources or instrument resolutions, these techniques can only obtain information on the overall site occupation ratio. The precise determination of specific atomic occupation and short-range order is still very rare, which extremely impedes the understanding of the correlation between the chemistry, micro-structure and the magnetic properties of M-type hexaferrites for further improvement in their performances.

M-type strontium hexaferrite $\left(\mathrm{SrFe}_{12} \mathrm{O}_{19}, \mathrm{SFO}\right)$ is one of the most important members in the M-type hexaferrite family, which has a magnetoplumbite structure with the $P 6_{3} / \mathrm{mmc}$ space group. Its hexagonal unit cell structure containing 64 
a
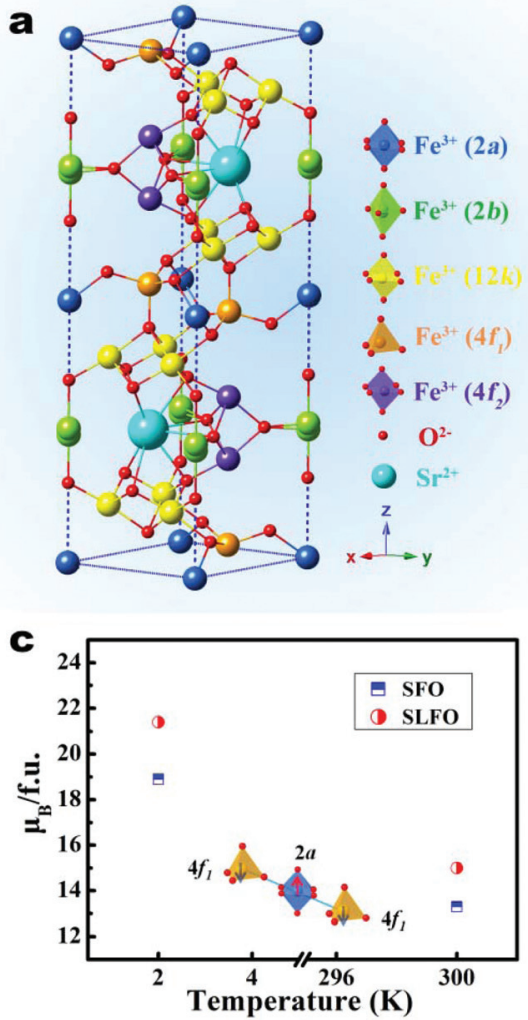

b
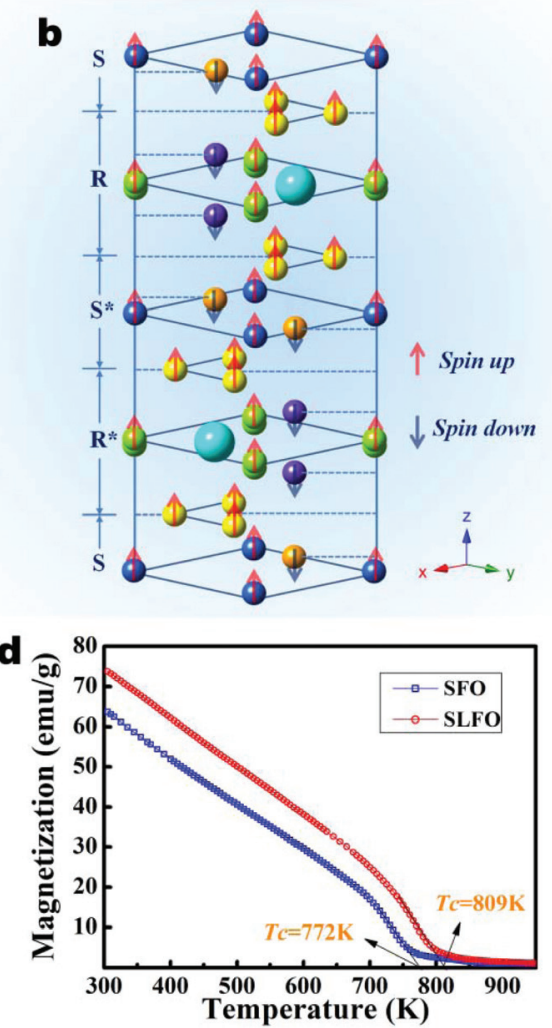

Fig. 1 Schematic of the crystal structure and ferrimagnetic ordering of magnetoplumbite SFO in a unit cell and magnetic characterization. (a) Crystal structure of $\mathrm{SrFe}_{12} \mathrm{O}_{19}$ can be described as RSR* $\mathrm{S}^{*}$ stacking sequence (left) and each unit cell contains 64 ions located at specific crystallographic sites. Sr cations and $\mathrm{O}$ anions are depicted as cyan and red spheres, respectively. Trivalent $\mathrm{Fe}^{3+}$ cations occupying five distinct crystallographic sites, including tetrahedral $\left(4 \mathrm{f}_{1}\right)$, octahedral $\left(2 \mathrm{a}, 12 \mathrm{k}\right.$ and $\left.4 \mathrm{f}_{2}\right)$, and trigonal-bipyramid $(2 \mathrm{~b})$ sites, are depicted as various coloured spheres, and their corresponding polyhedral sites are listed on the right. (b) Red and black arrows represent the strength and direction of magnetic moments, showing three parallel (12k, $2 \mathrm{a}$ and $2 \mathrm{~b})$ and two antiparallel $\left(4 \mathrm{f}_{1}\right.$ and $\left.4 \mathrm{f}_{2}\right)$ sub-lattices. (c) Plot of saturation magnetization ( $\mu_{\mathrm{B}} / \mathrm{f}$.u.) vs. temperature. (d) Temperature dependence of the magnetization measured at $3 \mathrm{~T}$ in the temperature range of $300-950 \mathrm{~K}$ to determine the Curie temperature.

ions can be described as the sequence $\mathrm{R} \mathrm{S} \mathrm{R}^{*} \mathrm{~S}^{*}$ spinel $(\mathrm{S}=$ $\left.\mathrm{Fe}_{6} \mathrm{O}_{8}{ }^{2+}\right)$ and hexagonal $\left(\mathrm{R}=\mathrm{SrFe}_{6} \mathrm{O}_{11}{ }^{2-}\right)$ layers, where the $\mathrm{S}^{*}$ and $\mathrm{R}^{*}$ blocks are axially symmetric around the hexagonal axis-c by $180^{\circ}$ (Fig. 1a). The magnetic cations of 24 trivalent $\mathrm{Fe}^{3+}$ are distributed on five different crystallographic sites, including one tetrahedral $\left(4 \mathrm{f}_{1}\right)$, three octahedral $(2 \mathrm{a}, 12 \mathrm{k}$ and $4 \mathrm{f}_{2}$ ) and one trigonal-bipyramid (2b) site. In contrast, the 38 $\mathrm{O}^{2-}$ anions are close-packed with the two $\mathrm{Sr}^{2+}$ ions in the hexagonal layer, showing three parallel (majority spin sites $12 \mathrm{k}, 2 \mathrm{a}$ and $2 \mathrm{~b}$ ) and two anti-parallel (minority spin sites $4 \mathrm{f}_{1}$ and $4 \mathrm{f}_{2}$ ) sub-lattices ${ }^{17-19}$ (Fig. 1b). All the Fe sites are trivalent Fe ions with a magnetic moment of $5 \mu_{\mathrm{B}}$ and have a high spin configuration of $\mathrm{d}^{5}(S=5 / 2)$, which have a vanishing orbital momentum. The total saturation moment is $40 \mu_{\mathrm{B}}$ per unit cell $\left(20 \mu_{\mathrm{B}}\right.$ per formula unit).$^{15}$ The substitution of rare-earth $\mathrm{La}^{3+}$ can strongly influence the correlated magnetic performance. It has been reported that the magneto-crystalline anisotropy and coercive field of M-type strontium hexaferrite can be increased or reduced according to the concentrations of the substituted $\mathrm{La}^{3+}$ cations. ${ }^{20-23} \mathrm{Fe}$ or $\mathrm{Sr}$ lattice sites are argued to be the strong preference for the $\mathrm{La}^{3+}$ substitutions, which are thought to be responsible for the intrinsic physical mechanism of mag- netism modulation. Many efforts using the abovementioned techniques have been devoted to confirming the site preference of the substituted ions in M-type strontium hexaferrite. However, unequivocal evidence of the preferred sites of the dopant La atoms is still lacking. The significant progress in aberration-corrected scanning transmission electron microscopy (Cs-corrected STEM) equipped with electron energy-loss spectroscopy (EELS) and energy-dispersive X-ray analysis (EDX) has achieved a surprisingly high spatial resolution below $0.5 \AA$, which readily realizes direct imaging and electronic-state detection of individual atoms and even chemical bonds. Moreover, Cs-corrected STEM allows direct observation of atomic placeholders and distributions because it collects high-angle scattered transmitted electrons to form highresolution and atomic number contrast images and collects low-angle (11 to $22 \mathrm{mrad}$ ) scattered transmitted electrons to image light atoms.

In this work, we performed an atomic-level investigation on the dopants and their occupation sites in La-doped $\mathrm{SrFe}_{12} \mathrm{O}_{19}$ nanofibres using aberration-corrected scanning transmission electron microscopy (STEM). Our measurements revealed that the doped La cations prefer to occupy the Sr crystallographic 
site rather than the reported diverse $\mathrm{Fe}^{3+}$ sub-lattices. Atomiccolumn resolution elemental mappings via electron energy loss spectroscopy (EELS) were further performed to detect the electronic states of the ions, which disclosed their spin states. Based on the spin states of the cations, we found that the physical mechanism of the magnetism modulation originates from the changes in the spin states of the magnetic iron ions at the $4 \mathrm{f}_{1}$ tetrahedral sites and $2 \mathrm{a}$ octahedral sites. Density functional theory (DFT) calculations further confirmed our experimental observations.

\section{Results}

We prepared SFO nanofibres and La-doped SFO nanofibres (SLFO) via the electrospinning technique (Methods). Both types of nanofibres formed a single-particle-chain continuous morphology and had a homogeneous chemical composition (ESI Fig. S1†). Individual particles on the SFO and SLFO nanofibres were found to be single crystal and have a magnetoplumbite structure (ESI Fig. S2 $\dagger$ ). The quantitative chemical analysis showed that SLFO had a composition of $\mathrm{Sr}_{0.9} \mathrm{La}_{0.1} \mathrm{Fe}_{12} \mathrm{O}_{19}$, indicating that $10 \%$ of the Sr ions were substituted by La ions. The magnetic properties of the SLFO and SFO nanofibres were characterised via SQUID and PPMS. The magnetic-field dependence of the magnetization of the SLFO and SFO nanofibres at $300 \mathrm{~K}$ and $2 \mathrm{~K}$ is shown in ESI Fig. S3. $\dagger$ The quantitative analysis showed that the saturation magnetization $\left(M_{\mathrm{s}}\right)$ and coercivity $\left(H_{\mathrm{c}}\right)$ of the SLFO nanofibres at $300 \mathrm{~K}$ and $2 \mathrm{~K}$ are $15 \mu_{\mathrm{B}} /$ f.u. (79.4 $\mathrm{emu} \mathrm{g}^{-1}$ ) and $6.70 \mathrm{kOe}$, and $21.4 \mu_{\mathrm{B}} /$ f.u. (113 $\mathrm{emu}^{-1}$ ) and $6.10 \mathrm{kOe}$, respectively. In comparison with the pure SFO nanofibres with $70.4 \mathrm{emu} \mathrm{g}^{-1}\left(13.3 \mu_{\mathrm{B}} /\right.$ f.u. $)$, $6.47 \mathrm{kOe}$ at $300 \mathrm{~K}$ and $99 \mathrm{emu} \mathrm{g}^{-1}\left(18.8 \mu_{\mathrm{B}} /\right.$ f.u. $), 5.71 \mathrm{kOe}$ at $2 \mathrm{~K}$, the magnetic moments respectively increased by $2.6 \mu_{\mathrm{B}} / \mathrm{f}$.u. at $2 \mathrm{~K}$ and $1.7 \mu_{\mathrm{B}} /$ f.u. at $300 \mathrm{~K}$, as shown in Fig. 1c, when the lanthanum was doped in the strontium hexaferrite. The coercivity slightly increased from $6.47 \mathrm{kOe}$ (SFO) to $6.70 \mathrm{kOe}$ (SLFO) at $300 \mathrm{~K}$ and $6.10 \mathrm{kOe}$ (SFO) to $5.71 \mathrm{kOe}$ (SLFO) at $2 \mathrm{~K}$. The increase in magnetization, $M_{\mathrm{s}}$, and coercivity, $H_{\mathrm{c}}$, should be associated with the substitution of $\mathrm{Sr}^{2+}$ cations by $\mathrm{La}^{3+}$ cations, which includes the occupation sites, canted spin structure and superexchange interactions between the cations and $\mathrm{O}^{2-}$ anions. ${ }^{26}$ Upon the substitution of rare-earth $\mathrm{La}^{3+}$ (1.22 $\AA$ radius) for $\mathrm{Sr}^{2+}$ (1.32 $\AA$ radius, larger than that of the $\mathrm{La}^{3+}$ ion) cations, the lattice constant $c$ shrinks compared with the undoped strontium ferrite and the $\mathrm{Fe}-\mathrm{O}$ distance parallel to the $c$-axis decreased, which facilitated the superexchange interaction. ${ }^{24}$ As shown in Fig. 1a, the crystal structure of M-type strontium hexaferrite contains two types of blocks with different symmetries, hexagonal packing R-block and cubic symmetry of spinel S-blocks, in which the S-blocks contain octahedral and tetrahedral interstitials sites. According to the Mössbauer studies, the change in the $\mathrm{Fe}^{3+}$ (high spin) valence state to the $\mathrm{Fe}^{2+}$ (low spin) state occurred at the 2a octahedral site (S-block) with higher symmetry to maintain electrical neutrality when the $\mathrm{Sr}^{2+}$ site was occupied by $\mathrm{La}^{3+} \cdot{ }^{25}$ In this case, according to the formula of net magnetization in hexaferrites:

$$
M=M_{12 \mathrm{k} \uparrow}+M_{2 \mathrm{a} \uparrow}+M_{2 \mathrm{~b} \uparrow}-\left(M_{4 \mathrm{f}_{1} \downarrow}+M_{4 \mathrm{f}_{2} \downarrow}\right)
$$

electron transfer between $\mathrm{Fe}^{3+}\left(5 \mu_{\mathrm{B}}\right)$ and $\mathrm{Fe}^{2+}\left(4 \mu_{\mathrm{B}}\right)$ at the $2 \mathrm{a}$ sites (majority sites) can eliminate a positive contribution, and hence reduce the total magnetic moment. However, the different cationic radii of $\mathrm{Fe}^{3+}(0.67 \AA)$ and $\mathrm{Fe}^{2+}(0.83 \AA)$ can cause deviations in the lattice sites and local strain, which may cause the nearest neighbor $4 \mathrm{f}_{1}$ sites to become disordered and modify their local electronic states. Local strain may also result in a canted spin structure with non-collinear magnetic order. ${ }^{26}$ Meanwhile, the $\mathrm{Fe}^{2+}$ cations at the 2a sites may migrate to the nearest neighbor $4 \mathrm{f}_{1}$ sites in the S-block (cubic symmetry), which causes a reduction in the magnetic moments of the $4 f_{1}$ sites. According to eqn (1), the total magnetic moment is correspondingly enhanced. ${ }^{23}$ The slight increase in coercivity is mainly attributed to the positive contribution of the effective anisotropy constant, which is related to transformation of $\mathrm{Fe}^{3+}$ to $\mathrm{Fe}^{2+}$ at the $2 \mathrm{a}$ octahedral sites. ${ }^{21}$

The Curie temperatures $\left(T_{\mathrm{c}}\right)$ of the SLFO and SFO nanofibres were also characterised in the temperature range of $300 \mathrm{~K}-1000 \mathrm{~K}$ at an external field of $3 \mathrm{~T}$ (Fig. 1d). $T_{\mathrm{c}}$ was measured to be $809 \mathrm{~K}$ when the lanthanum was doped, which is obviously higher than the value of $772 \mathrm{~K}$ for the SFO nanofibres. As mentioned above, the presence of $\mathrm{La}^{3+}$ in strontium ferrite leads to a contraction of the $c$-axis, which contributes to improving the strength of the $\mathrm{Fe}^{3+}-\mathrm{O}^{2-}-\mathrm{Fe}^{3+}$ super-exchange interaction. This increase in Curie temperature in the SLFO nanofibres is also attributed to the increase in super-exchange interactions. $^{27}$

To verify the correlation between micro-structure and magnetic properties, the ion distributions of the individual SFO and SLFO single-particles in the nanofibres were first imaged with atomic-resolution via aberration-corrected STEM. Fig. 2ac show three relevant atomic resolution HAADF-STEM images of the SFO nanofibres projected from the [001], [110] and [210] orientations. It is known that the contrast of HAADF-STEM images directly depends on the atomic number, $Z\left(\sim Z^{1.7}\right.$ dependence). In SFO, the atomic number of strontium, iron and oxygen is 38, 26 and 8, respectively. The relatively large differences among the $Z$ numbers significantly facilitate the formation of image contrast. As seen in the three-dimensional (3D) model of the SFO unit cell (Fig. 2d), there are four representative atomic columns along the [001] oriented SFO, including pure $\mathrm{Fe}$ atomic column numbered as $1, \mathrm{Fe}-\mathrm{O}$ mixed atomic column numbered as $2, \mathrm{Sr}-\mathrm{Fe}$ mixed atomic column numbered as 3 and pure $\mathrm{O}$ atomic column numbered as yellow 4 . It can be seen in Fig. 2a that the contrast of the atomic columns 1-3 is well matched with their effective $Z$ number except that column 4 for pure oxygen is not visible due to the low Rutherford scattering probability. In addition, the Sr atoms are easily distinguished from the others due to their much larger size than that of Fe. When the specimen was tilted to the [110] crystal axis, each atomic column contained only one element, 

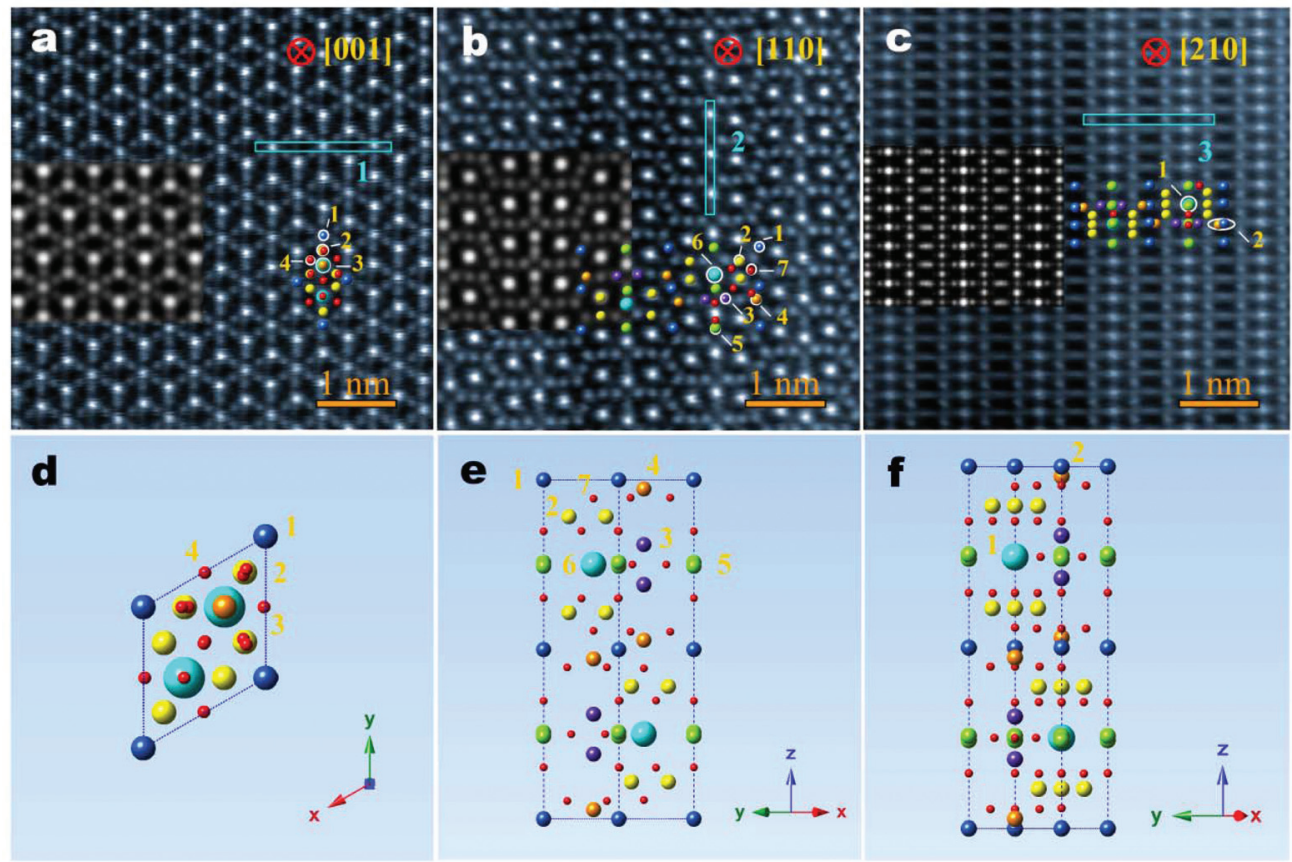

e
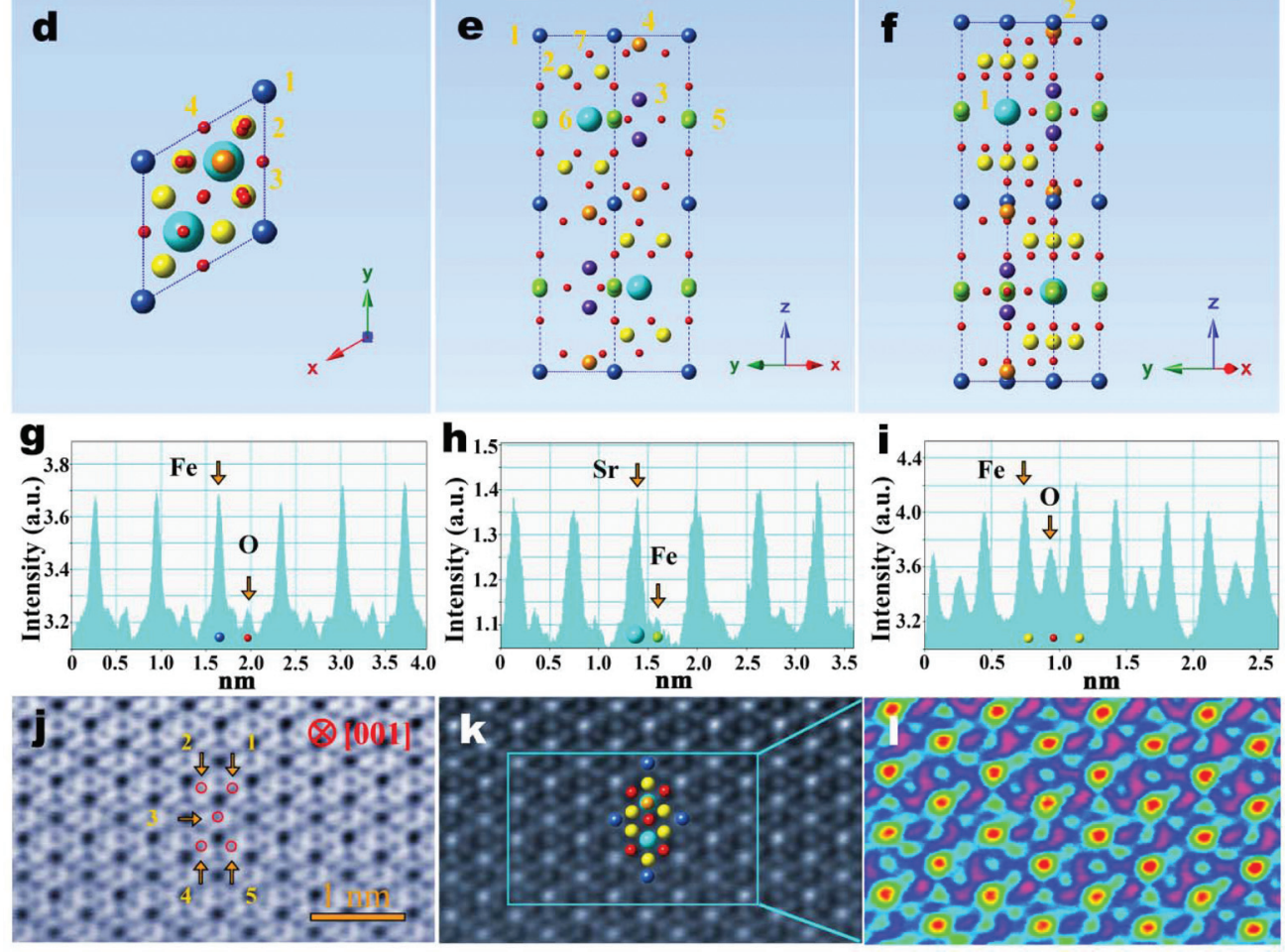

Fig. 2 Atomically resolved ion distributions of the M-type $\mathrm{SrFe}_{12} \mathrm{O}_{19}$ directly imaged by aberration-corrected STEM. (a-c) HAADF-STEM images taken along the [001], [110] and [210] orientations, respectively. Middle-left insets show the corresponding theoretical STEM images simulated using the QSTEM software, indicating a good match with the three experimental HAADF images. (d-f) Perspective views of the unit cell along the [001], [110] and [210] orientations, respectively. ( $\mathrm{g}-\mathrm{i}$ ) Line intensity profiles for the $\mathrm{Fe}, \mathrm{Sr}$ and $\mathrm{O}$ atomic columns along the rectangles numbered ' 1 ', ' 2 ' and ' 3 ' in (a)-(c), respectively. (j) ABF images of the SFO nanofibres viewed along the [001] zone axis, in which the red circles represent the $O$ anions labelled as 1, 2, 3, 4, and 5. (k) Contrast-reversed image of the ABF image (j). (l) Magnified micrograph of the area marked by blue rectangle in (k).

which provides an ideal situation to observe the ion distributions in SFO. The geometric sizes, positions and contrasts of the individual atomic columns in Fig. $2 \mathrm{~b}$ perfectly demonstrate that the SFO particles have an ideal magnetoplumbite structure, which matches well with the ion occupations and distributions illustrated by the 3D model of the SFO unit cell with the [110] orientation in Fig. 2e. In contrast, the [210] orientation (Fig. 2c) clearly shows that the ellipsoidal atom cluster marked by " 2 " is composed of three overlapping atomic columns, including two $\mathrm{Fe}$ atomic columns and an invisible $\mathrm{O}$ atomic column, matching well with the SFO 3D model with the [210] projection (Fig. 2f). The intensity profiles (Fig. 2g-i) of the three rectangles marked as blue numbers 1, 2 and 3 in Fig. $2 \mathrm{a}-\mathrm{c}$ further reveal the site positions and ordering of the
Sr, Fe and $\mathrm{O}$ atomic columns (see ESI Fig. $\mathrm{S} 4 \uparrow$ for the Fe and $\mathrm{O}$ columns), respectively. To precisely determine the occupation positions of the oxygen atoms, the $\mathrm{ABF}$ technique was employed to view the image of $\mathrm{O}$ atoms. Fig. $2 \mathrm{j}$ shows a relevant $A B F$ image of the same SFO specimen with the [001] orientation, which demonstrates that the mixed $\mathrm{Sr}-\mathrm{Fe}$ atomic columns are surrounded by five $\mathrm{O}$ atomic columns, as denoted by red circles with numbers $1-5$, in a unit cell, which form two symmetric triangles. The inverted contrast and magnified ABF images are shown in Fig. $2 \mathrm{k}$ and l, respectively, further indicating the precise lattice sites of the $\mathrm{O}$ atoms. The theoretical STEM images of SFO with an ideal magnetoplumbite structure in the left-middle insets of Fig. 2a-c were also simulated using QSTEM, showing the same placeholders. It is evident that the 
simulated images agree well with the experimental images, such as the ordering and contrast of the $\mathrm{Sr}$ and $\mathrm{Fe}$ cations along the three crystal orientations. Thus, the results show that the Cs-corrected STEM technique can reliably observe the specific atomic occupations and short-range order of the metallic cations and $\mathrm{O}$ anions in M-type hexaferrites.

We then imaged the specific substitution sites of the dopant La atoms in the SLFO nanofibres and analysed the chemical shifts of the composite elements. Fig. 3a shows the HAADF-STEM atomic image of the SLFO nanofibres, which is projected along the [110] orientation. In comparison with above atomic STEM image of pure SFO (Fig. 2a), this image reveals that the SLFO nanofibres retain the magnetoplumbite crystal structure and the La substitutions occur only in the $\mathrm{Sr}$ atomic columns. Fig. $3 \mathrm{~b}$ and $\mathrm{c}$ show the two corresponding $\mathrm{ABF}$ and contrast-reversed $\mathrm{ABF}$ images, revealing that the pure $\mathrm{Fe}$ atomic columns are surrounded by four pure $\mathrm{O}$ atomic columns, guided by the four red circles $1-4$. The four $\mathrm{O}$ atomic columns form a rectangle, demonstrating $\mathrm{O}^{2-}$ placeholders in the magnetoplumbite structure, which is consistent with the undoped SFO results. We also performed EDX atomic elemental mappings to verify the accurate sites of the substituted La ions. As shown in Fig. 3d-g, the result clearly discloses that the $\mathrm{La}^{3+}$ cations only randomly substituted for the $\mathrm{Sr}^{2+}$ sites rather than any of five $\mathrm{Fe}^{3+}$ distinct sub-lattices. The other atomic-resolution HAADF-STEM images and EDX atomic elemental mappings taken along the [001] and [210] orientations, as shown in ESI Fig. S5, $\dagger$ further verify our above observations that the SLFO nanofibres retained the magnetoplumbite structure and the La cations only replaced the $\mathrm{Sr}$ cations.

To further verify the preferred sites of the substitutional La ions, atomic-resolution dual-EELS elemental mappings were employed. Fig. 3h-k show the corresponding mappings of the oxygen $\mathrm{K}$ edges, iron $\mathrm{L}_{2,3}$ edges, lanthanum $\mathrm{M}_{4,5}$ edges and the combined mapping of these elements. The distribution of $\mathrm{La}$ (Fig. 3j) provides additional confirmation that the La doping only takes place at the lattice sites of the Sr cations, consistent with the EDX mapping result. Therefore, it can be concluded that the EELS elemental mapping analysis further confirms that the site preference of the doped La cations is the Sr sites and the distribution of the four ions ( $\mathrm{Sr}, \mathrm{La} \mathrm{Fe}$ and $\mathrm{O}$ ) in the SLFO nanofibres still has a magnetoplumbite structure.

The La substitution for the Sr ions should obviously result in a change in the electronic states of ions neighboring the La ions to achieve a new balance of electronic charge within the
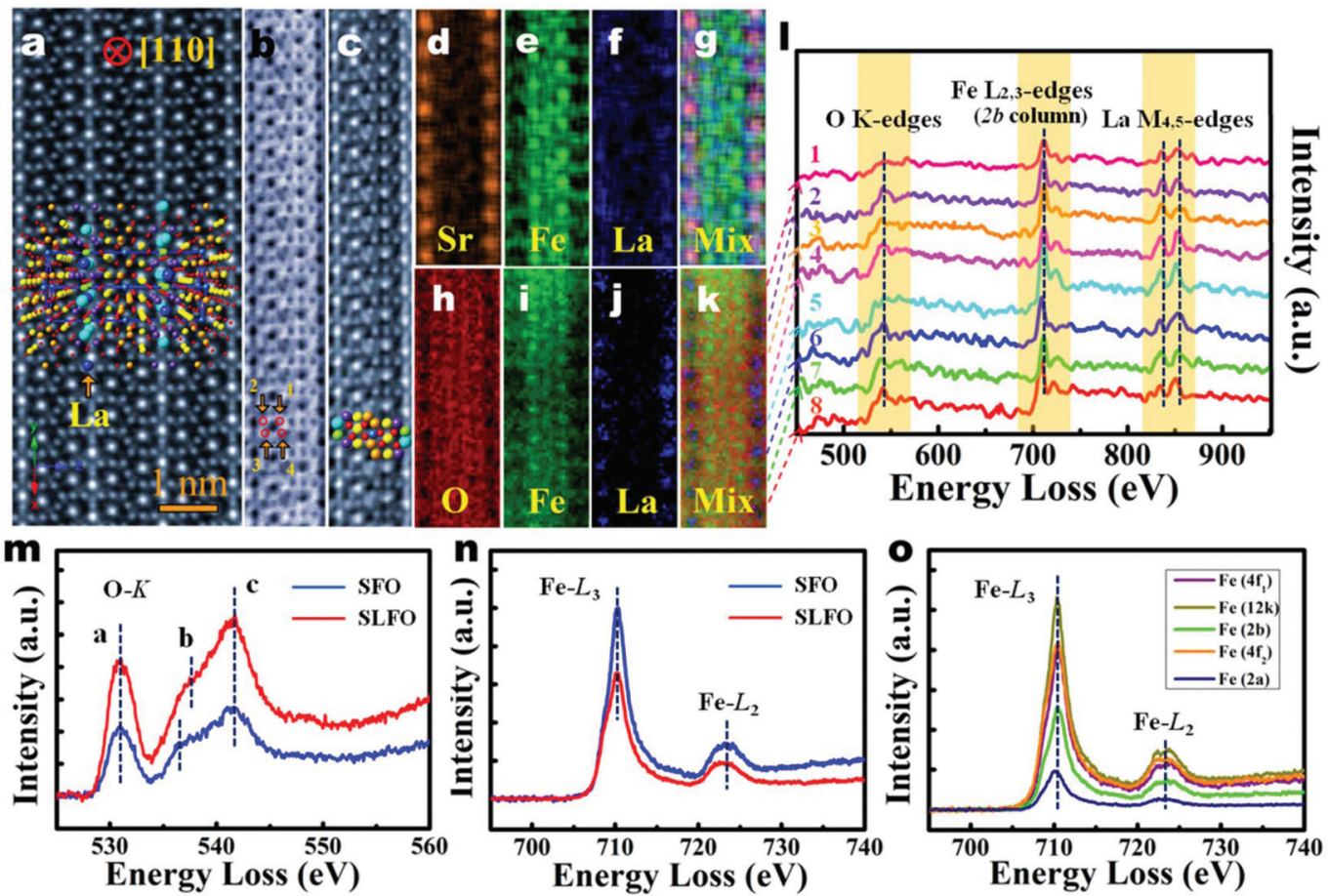

Fig. 3 La-Cation substitutional and valence state analysis of the $\mathrm{Sr}_{0.9} \mathrm{La}_{0.1} \mathrm{Fe}_{12} \mathrm{O}_{19}$ nanofibres. (a) Atomic-resolution HADDF-STEM image viewed from the [110] orientation. Insets: Perspective view of the [110] oriented cell showing the distribution of atomic columns. (b) Corresponding ABF micrograph of the SLFO nanofibres, in which the red circles represent $O$ anions numbered as 1, 2, 3, and 4. (c) Contrast-reversed image of the ABF micrograph. (d-g) EDX atomic-scale mappings of $\mathrm{Sr}$, Fe, and La elements and their mixture, respectively. ( $\mathrm{h}-\mathrm{k}$ ) Atomic-resolution dual-EELS mappings of oxygen ( $\mathrm{K}$ edge), iron ( $\mathrm{L}_{2,3}$ edge) and lanthanum $\left(\mathrm{M}_{4,5}\right.$ edge) and their colorized mapping projected along the [110] orientation, respectively. (l) Full EELS spectra of the eight La-Sr mixed atom columns extracted from the right of the spectrum image in (k). ( $m-n)$ Red and blue curves showing the $\mathrm{O} \mathrm{K}$-edge and $\mathrm{Fe} \mathrm{L}_{2,3}$-edge average EELS spectra of the SFO and SLFO nanofibres detected from about 15 atomic columns, respectively. (o) EELS line scans of $F e L_{2,3}$-edge at the 2a, 2b, 12k, $4 f_{1}$ and $4 f_{2}$ crystallographic sties, with peak positions of $710.09 \mathrm{eV}, 710.22 \mathrm{eV}, 710.32 \mathrm{eV}, 710.18$ $\mathrm{eV}$ and $710.30 \mathrm{eV} \mathrm{L}_{3}$, respectively. The error bar of each peak shift is $\pm 0.035 \mathrm{eV}$. 
La-doped SFO unit cell, which will naturally lead to a new magnetic state in the La-doped SFO. Therefore, obtaining detailed information on the electronic states of the ions can pave the way to better understand the mechanism of the modification of magnetic properties in doped SFO. Fig. 31 shows the EELS full spectra of the eight La-Sr mixed atom columns extracted from the right of spectrum image in Fig. $3 \mathrm{k}$, in which backgrounds were subtracted. These spectra indicate that the eight sites possess rich lanthanum elements with less oxygen, which is consistent with the theoretical occupation of atoms and atomic substitutions. The iron element signal originates from the neighbouring $\mathrm{Fe}$ atomic columns of the $2 \mathrm{~b}$ sites via the skirting effect ${ }^{28}$ of the incident electron beam because the $2 b$ Fe sites are too close to the $\mathrm{La}-\mathrm{Sr}$ sites under the [110] projected orientation. The Sr signal was not detected because its peak position is located at $1940 \mathrm{eV}$, which is close to the limitation of the EELS technique. Further detailed investigations on the electronic states were carried out using a smaller beam dispersion (0.05 eV per pixel). The red and blue curves in Fig. $3 \mathrm{~m}-\mathrm{n}$ show the averaged EELS spectra of the SFO and SLFO nanofibres based on the $\mathrm{O}$ K-edge and $\mathrm{Fe} \mathrm{L}_{2,3}$-edge mappings collected from about 15 atomic columns. The corresponding full spectra can be seen in ESI Fig. S6. $\dagger$ The three peaks of the $\mathrm{O}-\mathrm{K}$ edge, labeled by a, b and c (Fig. $3 \mathrm{~m}$ ), clearly indicate the hybridizations of $\mathrm{O} 2 \mathrm{p}$ with $\mathrm{Fe} 3 \mathrm{~d}, \mathrm{Sr} 4 \mathrm{~d}$, and Fe 4sp for SFO, and with Fe 3d, La 5d, and Fe 4sp for SLFO, respectively. ${ }^{29-31}$ It can also be seen that the onset energy of the pre-peak in the O K-edge spectrum of the SLFO nanofibres (red curve) is slightly smaller than that of the SFO nanofibres. The overall Fe $\mathrm{L}_{2,3}$-edge of SLFO (710.22 eV, $\mathrm{L}_{3}$ peak) appeared to be shifted by $0.08 \mathrm{eV}$ to the low energy region in comparison with that of SFO $(710.30 \mathrm{eV})$, as shown in Fig. 3n. This may be ascribed to the fact that the substitution of $2+$ valence $\mathrm{Sr}$ ions by $3+$ valence La ions resulted in a transformation in the electronic state of the Fe ions, i.e. their valence changed from $\mathrm{Fe}^{3+}$ to $\mathrm{Fe}^{2+}$, to maintain electrical neutrality in the La-doped strontium hexaferrite. Therefore, it is not surprising that the main $\mathrm{Fe}-\mathrm{L}_{2,3}$ peaks shifted to lower energies, indicating a reduced valence state. The ratio of Fe $\mathrm{L}_{3} / \mathrm{L}_{2}$ is about $4.66( \pm 0.12)$ for SFO and $4.50( \pm 0.10)$ for SLFO, confirming the decrease in the Fe valence state in the SLFO nanofibres. Magnetically, the spin state of the Fe ions changed accordingly, that is, from the high spin state of $\mathrm{Fe}^{3+}(S=5 / 2)$ to the low spin state of $\mathrm{Fe}^{2+x}$, where $x<1(S=2)$. The oxidation state of the La ions is $3+$, which coincides well with the standard La EELS spectrum in the EELS Atlas, as seen in ESI Fig. S7b. $\dagger$ Since both La and Sr are non-magnetic, the modification of the magnetic properties of the SFO nanofibres after La-doping should originate from the change in the magnetic moments of the Fe ions in the crystal. We further characterised all five crystallographic sites of the Fe ions by scanning individual Fe atomic columns via the EELS line-scan technique (see the scanning positions in ESI Fig. S7a†). Fig. 3o shows the relevant curves of the $\mathrm{Fe} \mathrm{L}_{2,3}$-edge, which show the $710.09 \mathrm{eV}$ $\mathrm{L}_{3}$ peak of the $2 \mathrm{a}$ site, $710.22 \mathrm{eV}$ of the $2 \mathrm{~b}$ site, $710.32 \mathrm{eV}$ of the $12 \mathrm{k}$ site, $710.18 \mathrm{eV}$ of the $4 \mathrm{f}_{1}$ site, and $710.30 \mathrm{eV}$ of the $4 \mathrm{f}_{2}$ site.
The $2 \mathrm{a}$ and $4 \mathrm{f}_{1}$ Fe sites of SLFO exhibit obvious chemical shifts of $0.21 \mathrm{eV}$ and $0.12 \mathrm{eV}$, respectively, in comparison with that of the SFO nanofibres, whilst the valence states of the other Fe sites do not show this shift. This result unambiguously discloses that the modification of the magnetic properties in the La-doped SFO permanent magnet originates from the changes in the electron states of the $2 \mathrm{a}$ and $4 \mathrm{f}_{1}$ Fe crystallographic sites, which indicates a fundamental way to tailor the magnetic performance of M-type hexaferrites.

Density functional theory (DFT) calculations were employed to further verify the above correlation of the chemistry and microstructures with the magnetic performance of the SLFO nanofibres. Fig. 4 presents the spin density and density of state (DOS) of the SLFO and SFO nanofibres calculated using a $1 \times 1$ unit cell. The result shows that the magnetic moment increased to $41 \mu_{\mathrm{B}}$ per unit cell after La-doping in comparison with the $40 \mu_{\mathrm{B}}$ per unit cell for SFO. This enhancement showed the same trend as the experimental measurement $\left(2.6 \mu_{\mathrm{B}} / \mathrm{f}\right.$.u. increase at $2 \mathrm{~K}$ after La-doping). The large deviation originates from the large difference in La-doping concentration between the theoretical calculation and the experimental specimen. The intensity of the total density of states of the system near the Fermi level was slightly reduced and the conduction band moved towards the Fermi level by $0.7 \mathrm{eV}$ after La-doping (ESI Fig. S8†). The increase in magnetic moment is related to the structure distortions after La-doping. As shown in Fig. 4a, the lengths of the $\mathrm{Fe}-\mathrm{O}$ bonds marked by the dashed green ellipses changed from $0.225 \mathrm{~nm}$ to $0.252 \mathrm{~nm}$ and $0.209 \mathrm{~nm}$ after La-doping. The reduced bond length resulted in an enhanced interaction between the $\mathrm{Fe}$ atom and the $\mathrm{O}$ atom located at the dashed blue circle in Fig. 4b, which finally induces strong couplings between the Fe4 atom (corresponding to the pink sphere at the $4 \mathrm{f}_{1}$ crystallographic site in Fig. 4a) and its neighbouring four $\mathrm{O}$ atoms in the $\mathrm{FeO}_{4}$ tetrahedron (dashed black ellipse in Fig. 4b) via the $\mathrm{FeO}_{6}$ octahedron (dashed yellow ellipse in Fig. 4b). Comparing the DOSs of the $\mathrm{Fe} 4$ atom (illustrated as pink $\mathrm{Fe} 4$ in Fig. 4a) before and after La-doping (Fig. 4c and d), it can be found that the majority of d states of the $\mathrm{Fe} 4$ atom move up towards the Fermi level, whilst the minority d states move from the unoccupied states to the occupied states just below the Fermi level. The quantitative analysis shows that the magnetic moment of the $\mathrm{Fe} 4$ atom decreased from $-4.38 \mu_{\mathrm{B}}$ to $-3.79 \mu_{\mathrm{B}}$ (negative magnetic moment denotes spin down) after La-doping, which discloses the intrinsic reason for the enhancement in magnetization. Close inspection of Fig. $4 \mathrm{e}$ and $\mathrm{f}$ reveals that near the Fermi level, the minority $\mathrm{Fe} 4 \mathrm{~d}_{z^{2}}$ orbital strongly hybridized with its surrounding $\mathrm{O} \mathrm{p}_{z}$ orbital. The four $\mathrm{O}$ atoms surrounding the $\mathrm{Fe} 4$ atom at the $4 \mathrm{f}_{1}$ site also have a small contribution to the increase in magnetic moment (about $0.10 \mu_{\mathrm{B}}$ per atom), as shown in Fig. 4f. In contrast, the DOSs of the Fe atoms at the $2 \mathrm{a}$ crystallographic site, denoted as Fe9, indicate their majority d states move away from the Fermi level up by $0.3 \mathrm{eV}$ after La-doping (ESI Fig. S9†). However, their magnetic moments remain unchanged. Therefore, the theoretical results reveal that the changes in the spin states of the $\mathrm{Fe}^{3+}$ cations 

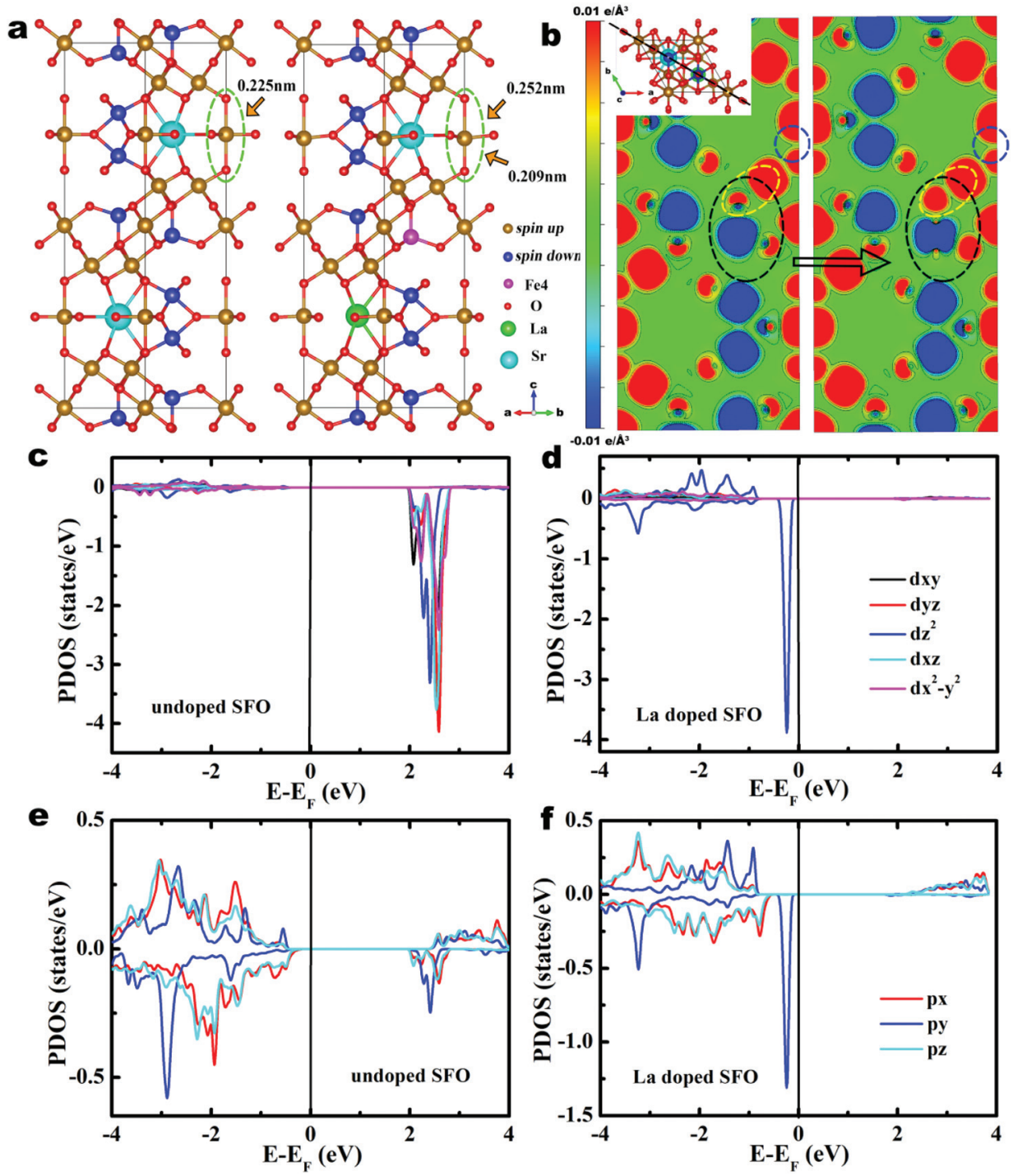

Fig. 4 Density functional theory calculations. (a) Left side: Ball \& stick model of SFO depicting Fe cations as gold spheres (spin up) and Fe cations as blue spheres (spin down), Sr cations in cyan, and $\mathrm{O}$ anions in red, which shows the same Fe ( $2 \mathrm{~b}$ site)-O band length along the $c$-axis. Right side: Ball $\&$ stick model of SLFO depicting the changed Fe4 cations in magenta and dopant La in green, which shows that the upper and lower bonds of the Fe (2b site)-O have different bond lengths along the c-axis. (b) Spin density maps of SFO and SLFO along the dash line shown in top-left inset of (b). Blue represents spin down and red is for spin up. Top-right inset shows a ball \& stick model depicting the orientation of the calculation. (c) $d$ orbitals of Fe4 atoms (at $4 f_{1}$ site) with different energy levels of SFO, (d) d orbitals of Fe4 atoms (at $4 f_{1}$ site) of SLFO with changed magnetic moments, (e) $p$ orbitals of $\mathrm{O}$ atoms around Fe 4 atoms of SFO and ( $f$ ) $p$ orbitals of $\mathrm{O}$ atoms around the Fe 4 atoms of SLFO.

located at the $4 \mathrm{f}_{1}$ tetrahedral sites result in an increase in magnetization and that for the 2 a octahedral sites contribute to the electronic charge balance after La-doping, well coinciding with the above experimental EELS and magnetic measurements.

\section{Conclusions}

In summary, we demonstrated the atomic-scale observation of the occupation sites and the occupancies of trace dopants in La-doped $\mathrm{SrFe}_{12} \mathrm{O}_{19}$ ferrite via aberration-corrected scanning transmission electron microscopy (STEM). The atomic STEM images, atomic-column resolution EDX elemental mappings and EELS mappings unequivocally revealed that the $\mathrm{La}^{3+}$ cations only randomly substitute for the $\mathrm{Sr}^{2+}$ sites rather than any of five distinct magnetic sub-lattices. The characterization of electronic states directly proved that the modification of the SLFO magnetization originates from the changes in the valence states located on the $4 \mathrm{f}_{1}$ and $2 \mathrm{a}$ crystallographic sites. The theoretical first-principles calculations further revealed that the La doping effectively affects the spin states of the $\mathrm{Fe}^{3+}$ cations located at the $4 \mathrm{f}_{1}$ tetrahedral sites and $2 \mathrm{a}$ octahedral sites, in which the $4 \mathrm{f}_{1}$ ions contribute to the enhancement in magnetization and the $2 \mathrm{a}$ ions maintain electrical neutrality. This work offers insights into the correlation between dopant atoms and magnetic properties in the complicated magneto- 
plumbite structure and provides guidance to tailor the magnetic performance of M-type hexaferrites.

\section{Methods}

\section{Sample synthesis}

Single-particle-chain M-type $\mathrm{SrFe}_{12} \mathrm{O}_{19} \quad$ (SFO) and $\mathrm{Sr}_{0.9} \mathrm{La}_{0.1} \mathrm{Fe}_{12} \mathrm{O}_{19}$ (SLFO) nanofibres were prepared via electrospinning. A typical precursor solution containing $0.1 \mathrm{mmol}$ strontium nitrate, $1.2 \mathrm{mmol}$ iron nitrite nonahydrate (0.1 mmol strontium nitrate, $1.19 \mathrm{mmol}$ iron nitrite nonahydrate and $0.01 \mathrm{mmol}$ lanthanum nitrate), $1 \mathrm{~mL} N, N$-dimethyl formamide (DMF), $0.3448 \mathrm{~g}$ of poly vinylpyrrolidone (PVP, $M_{\mathrm{w}}$ $\approx 1300000$ ), $0.5 \mathrm{~mL}$ deionized water and $2.5 \mathrm{~mL}$ ethanol alcohol was used. The electrospinning process was carried out at a DC voltage of $13 \mathrm{kV}$ with a gap of $5 \mathrm{~cm}$ from the needle tip to the collector and a feed rate of $0.4 \mathrm{~mL} \mathrm{~h}^{-1}$. The electrospun polymer composite nanofibres were heated at $200{ }^{\circ} \mathrm{C}$ for $2 \mathrm{~h}$ initially and then calcined at $880^{\circ} \mathrm{C}$ for $3 \mathrm{~h}$ at a heating rate of $1{ }^{\circ} \mathrm{C} \mathrm{min}^{-1}$ in air. The specimens were finally allowed to cool to room temperature at a rate of $1^{\circ} \mathrm{C} \mathrm{min}^{-1}$.

\section{Characterisation}

The atomic-scale crystalline structures of individual single-particle-chain $\mathrm{SrFe}_{12} \mathrm{O}_{19}$ and $\mathrm{Sr}_{1-x} \mathrm{La}_{x} \mathrm{Fe}_{12} \mathrm{O}_{19}(x=0.1)$ nanofibres were characterised using an aberration-corrected scanning transmission electron microscope (FEI Titan Cubed Themis G2 300, FEI, USA), operated at $300 \mathrm{kV}$ and equipped with a monochromator, Gatan image filter (GIF Quantum ER/965, Gatan, USA), EDX (Bruker EDX, Bruker, USA), Lorentz lens, Biprism and high-speed camera (Gatan K2 In situ, Gatan, USA). The morphology of the $\mathrm{SrFe}_{12} \mathrm{O}_{19}$ and $\mathrm{Sr}_{1-x} \mathrm{La}_{x} \mathrm{Fe}_{12} \mathrm{O}_{19}(x=0.1)$ nanofibres was characterised using a field-emission scanning electron microscope (Tescan MIRA 3 XMU, TESCAN, Czech). The phase compositions of the samples were determined using an X-ray diffractometer (XRD, Philips X'pert ProMPD, Netherlands) with $\mathrm{Cu} \mathrm{K} \alpha$ radiation. Magnetic properties were measured using a magnetic property measurement system (MPMS, SQUID-VSM, Quantum Design) and superconducting quantum interference magnetometer (SQUID, MPMS XL-7, UK).

\section{Simulations of atomic resolution STEM images}

The Quantitative TEM/STEM Simulations Package (QSTEM, Arizona State University, USA) was employed for quantitative simulations of the atomic-resolution STEM images. The simulation parameters were set-up by following the experimental conditions. The high voltage, Cc coefficients, Cs coefficient and defocus were $300 \mathrm{kV}, 1.2 \mathrm{~mm}, 0 \mathrm{~mm}$ and $0 \mathrm{~nm}$, respectively. The Nx, Ny and Nz of the unit cells were set to be $9 \times 9 \times$ 10 for slab calculations. The inner angle and outer angle of the HAADF detectors were $52 \mathrm{mrad}$ and $200 \mathrm{mrad}$, respectively. The semi-angle of the incident converged beam was $24.4 \mathrm{mrad}$. All simulated images used the same thickness of $42.6 \AA$ from the TEM measurement. The thermal diffuse scattering (TDS) runs were 30.

\section{Theoretical calculations}

First principles calculations were based on density functional theory (DFT) as implemented in the Vienna ab initio simulation package (VASP) code $\mathrm{c}^{32,33}$ with projector augmented wave (PAW) potentials and generalized gradient approximation (GGA) of Perdew, Burke, and Ernzerhof (PBE). The kinetic energy cutoff of the plane waves was set as $520 \mathrm{eV}$, and total energy was converged to $10^{-6} \mathrm{eV}$. The crystal geometry was optimized without symmetry constraints until all residual forces on each atom were less than $0.01 \mathrm{eV} \AA^{-1}$. A $1 \times 1$ unit cell was employed in all calculations, in which one $\mathrm{Sr}$ atom was substituted by an La atom. A $2 \times 2$ supercell in which one or two Sr atoms were substituted by La atoms was also checked for comparison, which showed the main results were nearly the same. To improve the description of the Fe $3 \mathrm{~d}$ electrons, the GGA+U method was employed to take into account strong correlation. $^{34}$ For SFO, reasonable agreement was reached once the effective Hubbard $U$ was equal to $7.0 \mathrm{eV}$ for Fe atoms. This value lies in the typical range, which has been proven to successfully describe the magnetic properties of $\operatorname{SrFe}_{12} \mathrm{O}_{19} \cdot{ }^{35}$

\section{Author contributions}

X.Z. and J.W.Z. contributed equally to this paper. X.Z. designed the experiments, prepared the specimens, characterised, analysed and interpreted all data, and wrote the paper. J.W.Z. carried out the aberration-corrected TEM characterisation. D.R.C. helped the specimen preparation. H.B.M. performed XRD measurements; X. D. and Q.Q.L. contributed EELS analysis. M.S.S. and D.S.X. contributed to the discussion of the paper and analysed the magnetic data. K.T. performed the DFT calculation and wrote the DFT part of the manuscript. X.X.Z. analysed the magnetic data and revised the English. Y.P. designed the experiments, provided overall guidance, supervised the TEM measurements, characterised, analysed and interpreted the data, and wrote the paper.

\section{Conflicts of interest}

The authors declare no competing financial interests.

\section{Acknowledgements}

This work was supported by National Natural Science Foundation of China (51771085, 51571104, 51801087, 51801088 and 11274145) and Open Project of Key Laboratory of Magnetism and Magnetic Materials of the Ministry of Education, Lanzhou University (LZUMMM2018003, LZUMMM2018012 and LZUMMM2019008).

\section{References}

1 C. J. Fennie and D. G. Schlom, Nat. Mater., 2010, 9, 787788. 
2 Y. Kitagawa, Y. Hiraoka, T. Honda, T. Ishikura, H. Nakamura and T. Kimura, Nat. Mater., 2010, 9, 797-802.

3 J. A. Kohn, D. W. Eckart and C. F. Cook Jr., Science, 1971, 172, 519-525.

4 S. E. Rowley, Y. S. Chai, S. P. Shen, Y. Sun, A. T. Jones, B. E. Watts and J. F. Scott, Sci. Rep., 2016, 6, 25724.

5 M. Shalaby, M. Peccianti, Y. Ozturk and R. A. Morandotti, Nat. Commun., 2013, 4, 1558.

6 M. Duerrschnabel, M. Yi, K. Uestuener, M. Liesegang, M. Katter, H. J. Kleebe, B. Xu, O. Gutfleisch and L. MolinaLunal, Nat. Commun., 2017, 8, 54.

7 I. V. Zavislyak, M. A. Popov and G. Srinivasan, Phys. Rev. B: Condens. Matter Mater. Phys., 2016, 94, 224419.

8 J. Park, Y. K. Hong, W. Lee, B. C. Choi and C. J. Choi, IEEE Magn. Lett., 2016, 7, 5500403.

9 D. Seifert, J. Topfer, F. Langenhorst, J. M. Le Breton, H. Chiron and L. Lechevallier, J. Magn. Magn. Mater., 2009, 321, 4045-4051.

10 J. M. Le Breton, J. Teillet, G. Wiesinger, A. Morel, F. Kools and P. Tenaud, IEEE Trans. Magn., 2002, 38, 2952-2954.

11 Z. J. Zhang, Z. L. Wang, B. C. Chakoumakos and J. S. Yin, J. Am. Chem. Soc., 1998, 120, 1800-1804.

12 D. Carta, M. F. Casula, A. Falqui, D. Loche, G. Mountjoy, C. Sangregorio and A. Corrias, J. Phys. Chem. C, 2009, 113, 8606-8615.

13 Y. Kobayashi, E. Oda, T. Nishiuchi and T. Nakagawa, J. Ceram. Soc. Jpn., 2011, 119, 285-290.

14 M. Ohtsuka, S. Muto, K. Tatsumi, Y. Kobayashi and T. Kawata, Microscopy, 2016, 65, 127-137.

15 H. Sakai, T. Hattori, Y. Tokunaga, S. Kambe, H. Ueda, Y. Tanioku, C. Michioka, K. Yoshimura, K. Takao, A. Shimoda, T. Waki, Y. Tabata and H. Nakamura, Phys. Rev. B: Condens. Matter Mater. Phys., 2018, 98, 064403.

16 A. Morel, J. M. Le Breton, J. Kreisel, G. Wiesinger, F. Kools and P. Tenaud, J. Magn. Magn. Mater., 2002, 242-245, 14051407.

17 C. M. Fang, F. Kools, R. Metselaar, G. de With and R. A. de Groot, J. Phys.: Condens. Matter, 2003, 15, 6229-6237.

18 J. Muller and A. Collomb, J. Magn. Magn. Mater., 1992, 103, 194-203.
19 Q. Li, J. Song, M. S. Múzquiz, F. Besenbacher, M. Christensen and M. D. Dong, Sci. Rep., 2016, 6, 25985.

20 H. Ueda, Y. Tanioku, C. Michioka and K. Yoshimura, Phys. Rev. B: Condens. Matter Mater. Phys., 2017, 95, 224421.

21 M. W. Pieper, F. Kools and A. Morel, Phys. Rev. B: Condens. Matter Mater. Phys., 2002, 65, 184402.

22 A. Thakur, R. R. Singh and P. B. Barman, J. Magn. Magn. Mater., 2013, 326, 35-40.

23 V. Dixit, C. N. Nandadasa, S. G. Kim, S. Kim, J. Park, Y. K. Hong, L. S. I. Liyanage and A. Moitra, J. Appl. Phys., 2015, 117, 243904.

24 M. Ghimire, S. Yoon, L. Wang, D. Neupane, J. Alam and S. R. Mishra, J. Magn. Magn. Mater., 2018, 454, 110-120.

25 M. Küpferling, R. Grossinger, M. W. Pieper, G. Wiesinger, H. Michor, C. Ritter and F. Kubel, Phys. Rev. B: Condens. Matter Mater. Phys., 2006, 73, 144408.

26 M. Mozaffari, A. Arab, M. H. Yousefi and J. Amighian, J. Magn. Magn. Mater., 2010, 322, 2670-2674.

27 F. van der Woude and G. A. Sawatzky, Phys. Rev. B: Solid State, 1971, 4, 3159-3165.

28 A. Zoukel, L. Khouchaf, J. D. Martino and D. Ruch, Micron, 2013, 44, 107-114.

29 M. Abbate, F. M. de Groot, J. C. Fuggle, A. Fujimori, O. Strebel, F. Lopez, M. Domke, G. Kaindl, G. A. Sawatzky, M. Takano, Y. Takeda, H. Eisaki and S. Uchida, Phys. Rev. B: Condens. Matter Mater. Phys., 1992, 46, 4511.

30 L. A. Grunes, R. D. Leapman, C. N. Wilker, R. Hoffmann and A. B. Kunz, Phys. Rev. B: Condens. Matter Mater. Phys., 1982, 25, 7157-7173.

31 A. Braun, D. Bayraktar, S. Erat, A. S. Harvey, D. Beckel, J. A. Purton, P. Holtappels, L. J. Gauckler and T. Graule, Appl. Phys. Lett., 2009, 94, 202102.

32 G. Kresse and J. Hafner, Phys. Rev. B: Condens. Matter Mater. Phys., 1993, 47, 558.

33 G. Kresse and J. Furthmuller, Phys. Rev. B: Condens. Matter Mater. Phys., 1996, 54, 11169.

34 S. L. Dudarev, G. A. Botton, S. Y. Savrasov, C. J. Humphreys and A. P. Sutton, Phys. Rev. B: Condens. Matter Mater. Phys., 1998, 57, 1505.

35 J. Park, Y. K. Hong, S. G. Kim, S. Kim, L. S. I. Liyanage, J. Lee, W. Lee, G. S. Abo, K. H. Hur and S. Y. An, J. Magn. Magn. Mater., 2014, 355, 1-6. 